Artigo Original

\title{
Severidades Ocupacionais associadas à inatividade física no lazer em trabalhadores
}

\author{
Camila Bosquiero Papini ${ }^{1}$ \\ Priscila Missaki Nakamura ${ }^{1,2}$ \\ Caroline de Oliveira Martins ${ }^{3}$ \\ Eduardo Kokubun ${ }^{1}$ \\ ${ }^{1}$ Instituto de Biociências. UNESP - Univ Estadual Paulista, Campus de Rio Claro, \\ Departamento de Educação Física, Núcleo de Atividade Física, Esporte e Saúde \\ (NAFES), Rio Claro, SP, Brasil \\ 2 Instituto de Biociências. UNESP - Univ Estadual Paulista, Campus de Rio Claro, \\ Departamento de Educação Física, Doutoramento em Ciências da Motricidade, Rio \\ Claro, SP, Brasil \\ ${ }^{3}$ Laboratório de Estudos e Pesquisas em Atividade Física e Saúde (LEPAFS), \\ Universidade Federal da Paraíba, João Pessoa, PB, Brasil
}

\begin{abstract}
Resumo: O objetivo do estudo foi verificar associações entre severidades ocupacionais e nível de atividade física no lazer em trabalhadores. Para isso participaram do estudo 200 trabalhadores ( 71 homens e 129 mulheres) do Município de Rio Claro e região. Foram avaliados o nível de atividade física no lazer (ativo ou inativo) e algumas severidades ocupacionais. A análise estatística revelou que o tempo da jornada diária de trabalho, realização de horas extra e intensidade do trabalho não foram associados à inatividade física no lazer em trabalhadores. Somente a disposição no fim da jornada de trabalho foi associada à inatividade física no lazer, mostrando que trabalhadores que tem alguma disposição depois do trabalho são mais ativos no tempo de lazer.
\end{abstract}

Palavras-chave: Trabalhadores. Nível de atividade física. Severidades ocupacionais.

\section{Occupational severities associated to leisure time physical inactivity level in workers}

\begin{abstract}
The aim of this study was to verify associations between occupational severities and leisure time physical inactivity level in workers. Two-hundred workers of Rio Claro County participated in this study. The leisure time physical activity (active or inactive) and some occupational severities were evaluated. The statistic analyses showed that working day, overtime work and occupational intensity had not been associated with leisure time physical inactivity in workers. Only the willingness at the end of the working day had been associated to the leisure time physical inactivity; showing that workers who leave their work with some willingness have more possibilities to be active in leisure time.
\end{abstract}

Key Words: Workers. Physical activity level. Occupational severities.

\section{Introdução}

Os benefícios da prática regular de Atividade Física (AF) para a saúde e/ou para prevenção de doenças têm sido cada vez mais ressaltados em diferentes meios de comunicação. Apesar de serem crescentes as evidências que um estilo de vida fisicamente ativo traga benefícios para a saúde a prevalência de inatividade física no lazer em adultos varia entre $59,8 \%$ a $88 \%$ no Brasil (GOMES et al., 2001; PITANGA e LESSA, 2005; VIGITEL, 2007). Vale salientar que na população de trabalhadores a prevalência de inatividade física no lazer é de 75\% (PAPINI, 2009).

Neste contexto, é necessário considerar o cotidiano das pessoas, muitas vezes vinculado a atividade ocupacional que deteriora a saúde, poucas opções de lazer determinadas por condições sócio-econômicas precárias e estruturas familiares voltadas unicamente para própria subsistência são apenas alguns dos aspectos que limitam a relação entre o indivíduo, exercício físico e saúde (MONTEIRO et al., 1998).

Além disso, a atividade ocupacional pode se tornar o campo ideal para o desenvolvimento de enfermidades e pode algumas vezes se constituir um fator limitante da expectativa de vida do ser humano. Os trabalhadores estão predispostos a sofrerem agravos por movimentos repetitivos ou lesões agudas, por indevida exposição ambiental, produtos tóxicos, ruídos, vibração, calor e frio 
excessivos, radiações, microrganismos bem como tensão, monotonia, estresse, etc (POLLOCK e WILMORE, 1993). O estudo realizado por Brandão et al. (2005) verificou que a jornada de trabalho, intensidade de trabalho, sexo feminino e a inatividade física estão associados com os distúrbios osteomusculares em trabalhadores.

Considerando que a grande parte da população passa a maior parte do seu tempo no local de trabalho, esse exerce grande influência na vida das pessoas. Todavia, de acordo com Barros e Nahas (2001), adultos em idade produtiva parecem estar expostos a barreiras e determinantes, ainda desconhecidos, que dificultam a adoção de comportamentos e de um estilo de vida mais favorável à manutenção da saúde e qualidade de vida.

Pensando na AF, existe a necessidade de investigar quais os determinantes que dificultam a adoção e manutenção de um estilo de vida fisicamente ativo em adultos e a partir disso criar estratégias e alternativas para favorecer uma mudança de comportamento. Ponderando o impacto que o trabalho exerce sobre a vida dos trabalhadores é necessário investigar se as características da atividade ocupacional estão associadas aos hábitos de vida dos trabalhadores. Alguns fatores como realização de horas extras, tempo da jornada diária do trabalho podem limitar o período de tempo livre do trabalhador, dificultando assim a prática de AF no lazer. Outros fatores ocupacionais como intensidade do trabalho podem influenciar a disposição ao fim do dia do trabalhador dificultando a realização de atividades mais ativas no tempo livre.

Assim, o objetivo desse estudo foi verificar se algumas severidades ocupacionais como realização de horas extras, tempo da jornada de trabalho, disposição ao final do trabalho e intensidade do trabalho estão associadas com a inatividade física no lazer em trabalhadores.

\section{Objetivo}

Verificar associações entre severidades ocupacionais e inatividade física no lazer em trabalhadores.

\section{Material e Métodos}

Participaram do estudo 200 trabalhadores, sendo 71 homens $(30,3 \pm 10,9$ anos $)$ e 129 mulheres (33,2 \pm 10 anos) de instituições públicas e privadas, com diferentes ocupações. Doze empresas da região de Rio Claro-SP foram visitadas e convidadas a participarem do estudo: houve uma recusa e onze concordâncias. Destas, quatro eram instituições públicas (hospital, escola, biblioteca e restaurante) e sete eram empresas privadas (indústrias, clínicas e escritórios). As empresas participantes foram classificadas em relação ao número de funcionários: a) 3 grandes empresas- mais de 500 trabalhadores; b) 5 médias empresas - 100 a 499 trabalhadores; c) 3 pequenas empresas - 20 a 99 trabalhadores. (SEBRAE, 1992). Esse estudo teve aprovação do Comitê de Ética em Pesquisa da Universidade Estadual Paulista (UNESP) de Rio Claro.

Cada empresa privada recebeu trinta questionários que foram entregues a um encarregado do departamento de Recursos Humanos, ficando responsável em distribuí-los aos trabalhadores. Para tanto, o mesmo recebeu informações a respeito dos objetivos do estudo e do preenchimento do instrumento de medida, bem como as implicações de sigilo sobre o nome da empresa, dos funcionários investigados e da utilização dos dados coletados apenas para fins de pesquisa. Para os trabalhadores das instituições públicas os questionários foram entregues por um dos autores do estudo. Os questionários foram preenchidos pelos próprios trabalhadores, aplicado no local de trabalho no período de junho e agosto de 2006.

Dos 30 questionários entregues a cada instituição privada, $81 \%$ (171) foram preenchidos corretamente e devolvidos e $100 \%$ (29) dos questionários entregues às instituições públicas foram preenchidos corretamente e devolvidos.

No procedimento de abordagem dos trabalhadores (privado e público), as seguintes instruções foram adotadas:

1. Apresentação dos objetivos do estudo;

2. Fornecimento e explicação a respeito do questionário;

3. Entrega e assinatura do Termo de Consentimento Livre e Esclarecido;

4. Entrega e preenchimento do questionário.

O questionário utilizado incluiu questões sobre sexo, idade, nível de AF no lazer e severidades ocupacionais.

Para avaliar o nível de AF foi empregado o IPAQ versão curta (International Physical Activity Questionnaire) traduzido para a língua 
portuguesa disponível no web site do Centro de Estudos do Laboratório de Aptidão Física de São Caetano do Sul/CELAFISCS (http://www.celafiscs.institucional.ws/65/questiona rios.html). A validade do IPAQ para o Brasil foi determinada em um estudo realizado por MATSUDO et al. (2001), com uma amostra de 257 homens e mulheres. A validade concorrente com o Computer Science Application (CSA) foi de 0,75 e a correlação de Spearman foi de rho $=$ $0,69-0,71: p<0.01$.

Para o presente estudo, os participantes foram perguntados somente sobre as AF no lazer. As orientações fornecidas pelo IPAQ foram utilizadas para o estabelecimento dos conceitos de AF de intensidades moderada e vigorosa. As AFs moderadas são aquelas que são realizadas por pelo menos 10 minutos, que provocam pequeno aumento na respiração ou frequência cardíaca. As AFs vigorosas são aquelas que induzem um grande aumento nessas mesmas variáveis.

A classificação do nível de AF no lazer do presente estudo foi calculada segundo 0 procedimento desenvolvido por Hallal (2003). O autor sugere multiplicar a duração da atividade vigorosa por dois e adicionar o tempo da atividade moderada e o tempo de caminhada. Através das informações obtidas pelo IPAQ, os trabalhadores foram classificados em ativos (realizam 150 minutos ou mais de AF por semana) e inativos (realizam menos de 150 minutos de AF por semana).

Para avaliar as severidades ocupacionais foi elaborado um questionário com interesse nas seguintes variáveis: a) jornada diária de trabalho (menos de 8 horas; 8 horas, mais de 8 horas); b) realização de hora extra (sim; não); c) disposição no fim da jornada de trabalho (disposto; cansado, mas com disposição; indisposto); d) intensidade do trabalho (leve; moderado; intenso). As seguintes definições da intensidade das tarefas ocupacionais foram adotadas: 1) leve (sentado, movimentos moderados com braços e troco- ex: datilografar; sentado, movimentos moderados com braços e pernas- ex: dirigir; de pé, trabalho leve, em máquina ou bancada, principalmente com os braços); 2) moderado (sentado, movimentos rigorosos com braços e pernas; de pé, trabalho leve/moderado em máquina ou bancada, com alguma movimentação; em movimento, trabalho moderado de levantar ou empurrar); 3) intenso (trabalho intermitente de levantar, empurrar ou arrastar pesos; trabalho fatigante).

A análise descritiva da amostra foi realizada por intermédio de Prevalência. Para a análise estatística foi realizado a Regressão de Poisson, obtendo a Razão de Prevalência (RP) e o Intervalo de Confiança (IC) de 95\%. Foi realizada análise bruta e ajustada por todas as variáveis. Como variável dependente foi utilizada inatividade física no lazer. As variáveis independentes utilizadas foram: sexo, idade, disposição ao fim da jornada de trabalho, horaextra, tempo diário da jornada de trabalho e intensidade do trabalho.

A análise foi realizada no programa STATA/SE 10.0. Em todos os testes estatísticos adotou-se como nível de significância $\mathrm{p}<0.05$.

\section{Resultados}

A figura 1 apresenta a classificação do nível de AF no lazer dos trabalhadores estudados.

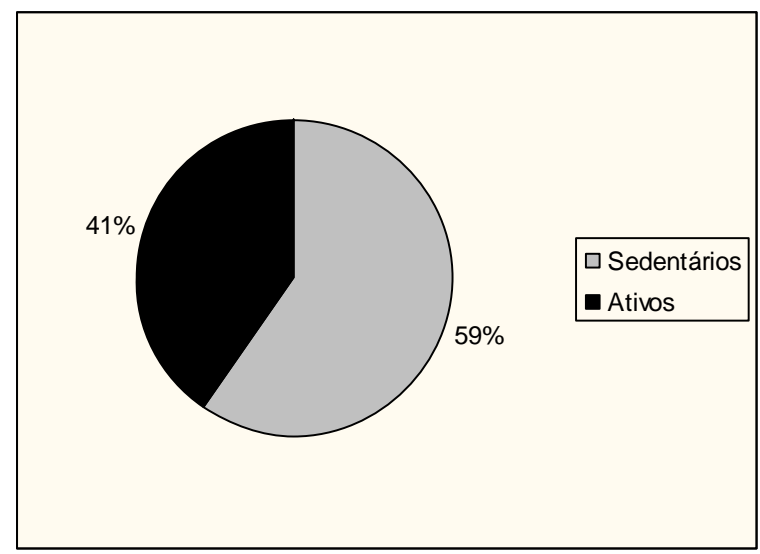

Legenda: Ativos = realizam 150 minutos ou mais de AF no lazer por semana; inativos = realizam menos de 150 minutos de AF no lazer por semana.

Figura 1. Classificação do nível de AF no lazer dos trabalhadores.

Dos trabalhadores participantes do estudo, $59 \%$ foram classificados como inativos (realizam menos de 150 minutos de AF no lazer por semana) e $41 \%$ foram classificados como ativos (realizam 150 minutos ou mais de AF no lazer por semana).

A tabela 1 informa a prevalência das respostas dos participantes estudados, relacionando a idade, disposição ao fim da jornada de trabalho, realização de hora extra, tempo diário da jornada de trabalho e intensidade do trabalho e também apresenta as associações entre as severidades ocupacionais e o nível de AF no lazer. 
A maioria dos trabalhadores participantes do estudo tem idade entre 20 a 29 anos (87\%) e entre 30 a 39 anos (55\%). Apenas $7.5 \%$ se sentem indisposto ao fim da jornada, sendo $1 \%$ de homens e $6.5 \%$ de mulheres. Dos participantes do presente estudo $30 \%$ realizam horas extras de trabalho. Com relação à jornada de trabalho, $44 \%$ dos trabalhadores trabalham 8 horas por dia e $48 \%$ trabalham mais de 8 horas por dia. Para a intensidade do trabalho, 58.5\% afirmam realizar trabalho leve, $33.5 \%$ trabalho de intensidade moderada e $8 \%$ afirmaram realizar trabalho intenso.

Tabela 1. Descrição da Amostra (n/\%); Razão de Prevalência (RP) e intervalos de confiança (IC95\%) para as variáveis independentes de acordo com o nível de AF lazer.

\begin{tabular}{|c|c|c|c|c|c|c|c|}
\hline \multirow[b]{3}{*}{ Variáveis } & \multirow[b]{3}{*}{ n (\%) } & \multicolumn{6}{|c|}{ Inatividade Física no Lazer } \\
\hline & & \multicolumn{3}{|c|}{ Bruta } & \multicolumn{3}{|c|}{ Ajustada** } \\
\hline & & $\mathbf{R P}$ & IC & $\mathbf{P}$ & $\mathbf{R P}$ & IC & $\mathbf{P}$ \\
\hline \multicolumn{8}{|l|}{ Sexo } \\
\hline Mulher & $129(64.5)$ & 1 & & & 1 & & \\
\hline \multicolumn{7}{|l|}{ Idade (anos) } & $0,043^{*}$ \\
\hline 15 a 19 & $12(6)$ & 1 & & & 1 & & \\
\hline 20 a 29 & $87(43.5)$ & 0,99 & $0,58-1,69$ & 0,978 & 0,86 & $0,52-1,41$ & 0,560 \\
\hline 30 a 39 & $55(27.5)$ & 1,14 & $0,66-1,95$ & 0,629 & 0,90 & $0,54-1,49$ & 0,694 \\
\hline 40 a 49 & $31(15.5)$ & 0,96 & $0,53-1,74$ & 0,913 & 0,80 & $0,45-1,48$ & 0,460 \\
\hline$\geq 50$ & $15(7.5)$ & 0,44 & $0,16-1,18$ & 0,106 & 0,38 & $0,15-0,96$ & $0,041^{*}$ \\
\hline \multicolumn{8}{|c|}{ Disposição ao fim da jornada de trabalho } \\
\hline Disposto & $51(25.5)$ & 1 & & & 1 & & \\
\hline Cansado & $134(67)$ & 1,66 & $1,15-2,40$ & $0,006^{*}$ & 1,51 & $1,05-2,17$ & $0,024^{*}$ \\
\hline Indisposto & $15(7.5)$ & 1,87 & $1.18-2,96$ & $0,008^{*}$ & 1,59 & $1,01-2,56$ & $0,050^{*}$ \\
\hline \multicolumn{8}{|l|}{ Hora-extra } \\
\hline Não & $139(69.5)$ & 1 & & & 1 & & \\
\hline Sim & $61(30.5)$ & 0,95 & $0,73-1,23$ & 0,718 & 0,97 & $0,74-1,28$ & 0,878 \\
\hline \multicolumn{8}{|c|}{ Tempo diário da jornada de trabalho (horas) } \\
\hline $8 \mathrm{~h}$ & $88(44)$ & 1 & & & 1 & & \\
\hline$<8$ & $96(48)$ & 0,85 & $0,51-1,40$ & 0,535 & 0,89 & $0,55-1,43$ & 0,645 \\
\hline$>8$ & $16(8)$ & 0,91 & $0,72-1,16$ & 0,472 & 0,93 & $0,73-1,20$ & 0,620 \\
\hline \multicolumn{8}{|c|}{ Intensidade do trabalho } \\
\hline Leve & $117(58.5)$ & 1 & & & 1 & & \\
\hline Moderado & $67(33.5)$ & 1,20 & $0,95-1,53$ & 0,123 & 1.13 & $0,89-1,45$ & 0,296 \\
\hline Vigoroso & $16(8)$ & 1,26 & $0,87-1,83$ & 0,213 & 1.16 & $0,76-1,72$ & 0,486 \\
\hline
\end{tabular}

De acordo com a análise ajustada, apresentada na tabela 1 , os trabalhadores do sexo masculino têm menor associação ( $R P=$ $0,74-\mathrm{IC}=0,56-0,99)$ com a inatividade física no lazer quando comparado com as mulheres. Com relação à idade, para a amostra estudada, a associação com a inatividade física no lazer é reduzida $(\mathrm{RP}=0,38-\mathrm{IC}=0,15-0,96)$ para os trabalhadores com 50 anos ou mais quando comparados com trabalhadores de 15 a 19 anos. Os trabalhadores que se sentem cansados e indispostos possuem associação positiva com a inatividade física no lazer $(R P=1,51-I C=1,05-$ 2,17 e $\mathrm{RP}=1,59-\quad \mathrm{IC}=1,01-2,56$ respectivamente) do que aqueles que se sentem dispostos ao final da jornada de trabalho. A realização de horas extras, o tempo diário da jornada de trabalho e a intensidade do trabalho não estão associados com a inatividade física no lazer. No entanto, podemos observar que há uma tendência em aumentar a razão de prevalência para associação com a inatividade física no lazer conforme aumenta a intensidade do trabalho.

\section{Discussão}

A prevalência de inatividade física no lazer em trabalhadores encontrada nesse estudo foi de $59 \%$, um número inferior quando comparado com um levantamento feito no Município de Rio ClaroSP que apontou que a prevalência de a inatividade física no lazer em trabalhadores é de $75 \%$ (PAPINI, 2009). O motivo dessa diferença se deve pela seleção da amostra de trabalhadores estudada. No presente estudo os trabalhadores foram selecionados por conveniência, a partir da facilidade de acesso em algumas empresas do Município de Rio Claro-SP e região. Já no levantamento realizado por Papini (2009) no 
mesmo Município a amostra de 864 trabalhadores foi selecionada de forma aleatória estratificada por setores censitários sugerindo uma melhor representatividade da população estudada.

Os trabalhadores do sexo masculino têm menor associação $(\mathrm{RP}=0,74-\mathrm{IC}=0,56-0,99)$ com a inatividade física no lazer quando comparado com as mulheres, sendo esse resultado apoiado com outros estudos encontrados na literatura. Em um estudo realizado por Oliveira (2000) com funcionários de uma empresa estatal, foi igualmente observado que os homens $(\mathrm{OR}=1)$ têm menor chance de serem inativos no lazer quando comparados com as mulheres $(O R=1,40-I C=1,05-1,82)$. No estudo realizado por Nunes e Barros (2004) em trabalhadores de diferentes ocupações (indústria, administração e prestação de serviços) foi observado que 0 sexo feminino esteve significativamente associado à prevalência de inatividade física no lazer $(\mathrm{OR}=4,28-\mathrm{IC}=1,74$ 10,64). Os mesmos autores consideram que as mulheres podem ser mais ativas fora do lazer (nas obrigações domésticas de limpeza, higiene pessoal e alimentação, que demandem esforço moderado ou mesmo vigoroso), conforme o padrão atribuído a elas culturalmente, o que levaria à opção por um estilo de vida mais inativo no lazer. Por fim, as mulheres podem preferir optar por atividades passivas, de intensidade leve durante o lazer. No estudo de Barros e Nahas (2001) ao avaliar o nível de AF no lazer de trabalhadores da indústria de Santa Catarina observaram que entre os trabalhadores considerados inativos no lazer, $67 \%$ eram mulheres e $33 \%$ eram homens. Uma possível explicação para tal constatação, que precisa ser mais bem estudada, está na dupla jornada das mulheres, que acumulam os afazeres domésticos e as tarefas ocupacionais dificultando assim a realização de AF no lazer.

No levantamento realizado com amostra representativa de trabalhadores $(n=864)$ da cidade de Rio Claro-SP, o sexo feminino também mostrou-se associado com a inatividade física no lazer (mulheres $\mathrm{RP}=1$ e homens $\mathrm{RP}=0,92-\mathrm{IC}=$ 0,85 - 0,99). Nesse mesmo levantamento foi observado que com 0 aumento da idade, aumenta-se o tempo de AF no lazer dos trabalhadores. (PAPINI, 2009). Esse resultado está de acordo com os achados do presente trabalho, onde foi constatado que a associação com a inatividade física no lazer é reduzida $(\mathrm{RP}=$ $0,38-\mathrm{IC}=0,15-0,96)$ para os trabalhadores com 50 anos ou mais quando comparados com trabalhadores de 15 a 19 anos (RP $=1)$. No entanto, alguns estudos mostram que para os trabalhadores em geral, quanto maior a faixaetária menor a chance do trabalhador se engajar em AF no lazer (SALLES-COSTA, 2003; NUNES; BARROS, 2004).

Não houve qualquer associação entre nível de AF no lazer em trabalhadores com a realização de horas extras e o tempo diário da jornada de trabalho. Apesar da análise estatística apontar uma tendência em aumentar a associação da inatividade física no lazer conforme aumenta-se a intensidade do trabalho (leve, moderado e vigoroso), esse não foi um resultado significativo. Esse mesmo resultado foi encontrado no levantamento realizado em Rio Claro-SP afirmando que não há qualquer associação entre nível de AF no trabalho e AF no lazer nos trabalhadores (PAPINI, 2009).

A discussão em torno da intensidade do trabalho como fator de risco para a inatividade física no horário de lazer é controversa na literatura. A relação inversa de inatividade física no trabalho e no lazer foi observada no estudo realizado por MONTEIRO et al. (1998), onde as pessoas classificadas como inativas apresentavam maior carga de trabalho. $O$ estudo de Pratt e Munshi (1994) também ressaltou que a alta prevalência de inatividade física no lazer em oito comunidades do estado de Nova lorque (EUA) estava associada a um nível mais alto de AF ocupacional, de transporte e de locomoção. Salmon et al. (2000), observaram o nível de AF no lazer, nas atividades ocupacionais e no domicílio, concluindo que a exposição do trabalhador de menor nível sócio-econômico aos esforços físicos contínuos de alta intensidade no trabalho e domicílio justificavam a maior prevalência de inatividade física durante o lazer nesse grupo. Porém, os resultados encontrados por Oldridge et al. (1983) apontavam a inatividade física no trabalho (ocupações de leve ou muito leve intensidade) como um importante fator para a adoção de hábitos sedentários também durante - lazer, fato agravado pelo processo de industrialização e mecanização da sociedade, que têm contribuído para a economia de esforço físico no quotidiano das pessoas. 
Quando consideramos a disposição no fim da jornada de trabalho, a análise estatística indicou que tanto os trabalhadores que se sentem cansados $(R P=1,51-I C=1,05-2,17)$ quantos aqueles que se sentem indispostos $(\mathrm{RP}=1,59$ $\mathrm{IC}=0,99-2,56)$ são mais inativos no lazer. Entre os trabalhadores que se sentem cansados $\mathrm{e}$ indispostos no fim da jornada de trabalho, a maioria é representada pelas mulheres $(46 \%$ e $6.5 \%$ respectivamente). Esse resultado pode esclarecer a associação entre inatividade física com o sexo feminino, já que a disposição no fim da jornada de trabalho também está associada à inatividade física no lazer.

Apesar da não associação direta entre nível de AF no lazer com realização de horas-extras, tempo diário da jornada de trabalho e a intensidade do trabalho, essas são severidades que podem influenciar a disposição do trabalhador ao fim do trabalhado, necessitando assim estudos mais detalhados sobre essa influência.

É importante ressaltar algumas limitações do presente trabalho: a) seleção de amostra por conveniência; b) utilização de delineamento transversal que dificulta a discussão sobre causa e efeito; c) realização de estudo em uma população específica de trabalhadores da região de Rio Claro/SP; d) utilização de questionários; e) falta de validação do instrumento utilizado para coletar os dados sobre severidades. Com relação às limitações desse estudo, não é possível estender os resultados encontrados para a população de trabalhadores em geral. No entanto esclarece algumas questões que estão associadas ou não à inatividade física no lazer entre trabalhadores, dando início às discussões e investigações mais aprofundadas sobre 0 assunto.

O ambiente de trabalho é um local que exerce grande influência na vida das pessoas, tanto pelo aspecto físico/ambiental, aspecto organizacional e aspecto social. Considerando que grande parte da população passa a maior parte do tempo no local de trabalho, esse pode ser o ponto de partida para a promoção da saúde dentro e fora do ambiente ocupacional (PAHO, 2001; MARTINS, 2008). A melhoria de condições de trabalho, cuidados médicos, bem como iniciativas das empresas em promover palestras e atividades relacionadas à promoção da saúde, inclusive implantando programas de ginástica laboral, deveriam ser estratégias adotadas para reduzir os trabalhadores a fatores de risco à saúde.

\section{Conclusão}

Das variáveis estudadas somente a disposição ao fim da jornada de trabalho foi associada ao nível de AF no lazer, sugerindo que os trabalhadores que se sentem dispostos ao fim da jornada de trabalho são aqueles que se engajam em AF no tempo de lazer, enquanto que os trabalhadores que se sentem cansados e indispostos ao fim da jornada de trabalho praticam atividades mais passivas no lazer.

\section{Referências}

BRANDÃO, A.G. et al. Sintomas de distúrbios osteomusculares em bancários de Pelotas e região: prevalência e fatores associados. Revista Brasileira de Epidemiologia, v.8, n.3, p.295-305, 2005.

BARROS, M.V; NAHAS, M.V. Comportamento de risco, auto-avaliação do nível de saúde e percepção de estresse entre trabalhadores de indústria. Revista de Saúde Pública, v. 35, p.554-63, 2001.

GOMES, V.B. et al. Atividade física em uma amostra probabilística da população do município do Rio de Janeiro. Caderno de Saúde Pública, v. 17, n. 4, p.969-976, 2001.

HALLAL, P.C. et al. Physical inactivity: prevalence and associated variables in Brazilian adults.

Medicine and Science in Sports and Exercise, v.35, n.11, p.1894-1900, 2003.

MARTINS, C.O. Programa de promoção da saúde do trabalhador. Jundiaí (SP): Fontoura, 2008.

MATSUDO, S. et al. Questionário internacional de atividade física (IPAQ): estudo de validade e reprodutibilidade no Brasil. Revista Brasileira de Atividade Física e Saúde, v.6, n.2, p.5-18, 2001.

MONTEIRO, H.L. et al. Fatores sócio-econômicos e ocupacionais e a prática de atividade física regular: estudo a partir de polícias militares em Bauru, São Paulo. Motriz, v. 4, n. 2, dez, 1998.

NUNES, J.O.M.; BARROS, J.F. Fatores de risco associados a prevalência de sedentarismo em trabalhadores da indústria e da Universidade de Brasília. Lecturas, educacion física y deportes, Buenos Aires, ano 10, n. 69, fev, 2004.

OLDRIGE, N.B. et al. Predictors of dropout from cardiac rehabilitation. Ontario Exercise Health 
Collaborative Study. American Journal

Cardiology, v. 51, p. 70-74, 1983.

OLIVEIRA, C.C.M. Atividade física de lazer e sua associação com variáveis demográficas e outros hábitos relacionados à saúde em funcionários de banco estatal. Dissertação de Mestrado. Rio de Janeiro: Escola Nacional de Saúde Pública, Fundação Oswaldo Cruz, 2000.

PAHO - PAN AMERICAN HEALTH ORGANIZATION. Regional plan on workers' health. PAHO: Washington, DC (USA), 2001.

PAPINI, C.B. Associações entre nível, oferta de atividade física no trabalho e atividade física de lazer. Dissertação apresentada ao Instituto de Biociências da Universidade Estadual Paulista para obtenção do título de Mestre em Ciências da Motricidade. Rio Claro, 2009.

PITANGA, F.J.G; LESSA, I. Prevalência e fatores associados ao sedentarismo no lazer em adultos. Caderno de Saúde Pública, v.21, n.3, p.870 877, mai-jun, 2005.

PRATT, M.; MUNSHI, O. A descriptive epidemiology of leisure time physical activity in eight New York state communities. Medicine and Science in Sports and Exercise (Supp), vol. 23, n. 4, S105, 1994.

POLLOCK, M.L.; WILMORE, J.H. Exercícios na saúde e na doença. São Paulo: Medsi, 1993.

SALLES-COSTA et al. Associação entre fatores sócio-demográficos e prática de atividade física de lazer no estudo Pró-Saúde. Caderno de Saúde Pública, v.19, n.4, p. 1095-1105, jul-ago, 2003.

SALMOM, B.A. et al. Leisure-time, occupational, and household physical activity among professional, skilled and less-skilled workers and homemakers. American Journal of Preventive Medicine, New York, v. 30, p. 191-199, março, 2000.

SEBRAE. Serviço Brasileiro de Apoio às Micro e Pequenas Empresas. Como abrir seu próprio negócio. Manual prático de iniciação empresarial. Brasília: SEBRAE, p.2 -12, 1992.

VIGITEL. Vigilância de fatores de risco e proteção para doenças crônicas por inquérito telefônico 2006. Ministério da Saúde: 2007.
Endereço:

Camila Bosquiero Papini

Avenida 4A, 814 Bela Vista

Rio Claro SP Brasil

13506-770

Telefone: (19)9211.5627 3557.83893526 .4307

e-mail: camila@papini.com.br

Recebido em: 30 de outubro de 2009.

Aceito em: 25 de maio de 2010.

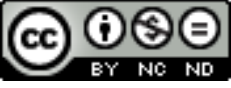

Motriz. Revista de Educação Física. UNESP, Rio Claro, SP, Brasil - elSSN: 1980-6574 - está licenciada sob Licenca Creative Commons 